\title{
Correlationship between Ki67, VEGF, and p53 and Hepatocellular Carcinoma Recurrence in Liver Transplant Patients
}

\author{
Xia Zhang, ${ }^{1}$ Zhixian Wu, ${ }^{1}$ Yonghai Peng, ${ }^{2}$ Dongliang Li, ${ }^{1}$ Yi Jiang, ${ }^{1}$ Fan Pan, ${ }^{3}$ Yi Li, \\ Yanhua Lai, ${ }^{5}$ Zhongyuan Cui $\mathbb{D}^{1},{ }^{1}$ and Kun Zhang ${ }^{6}{ }^{6}$ \\ ${ }^{1}$ Department of Hepatobiliary Disease, The 900th Hospital of the People's Liberation Army Joint Service Support Force \\ (Dongfang Hospital), Xiamen University, Fuzhou, Fujian, China \\ ${ }^{2}$ Department of Oncology, The 900th Hospital of the People's Liberation Army Joint Service Support Force (Dongfang Hospital), \\ Xiamen University, Fuzhou, Fujian, China \\ ${ }^{3}$ Department of Hepatobiliary Surgery, The 900th Hospital of the People's Liberation Army Joint Service Support Force, China \\ ${ }^{4}$ Department of Oncology, 920th Hospital of Joint Logistics Support Force, China \\ ${ }^{5}$ Department of Transplantation, People's Hospital of Guangxi Zhuang Autonomous Region, China \\ ${ }^{6}$ Department of Hepatobiliary Surgery, Xiang'an Hospital, Xiamen University, Xiamen, China
}

Correspondence should be addressed to Zhongyuan Cui; cuizhongyuan@stu.xmu.edu.cn and Kun Zhang; liliedzh@yeah.net

Received 26 November 2020; Revised 7 March 2021; Accepted 30 March 2021; Published 16 April 2021

Academic Editor: Junyan Tao

Copyright (c) 2021 Xia Zhang et al. This is an open access article distributed under the Creative Commons Attribution License, which permits unrestricted use, distribution, and reproduction in any medium, provided the original work is properly cited.

\begin{abstract}
Background and Aims. Patients with hepatocellular carcinoma (HCC) who undergo orthotopic liver transplantation (OLT) are at risk for posttransplant tumor recurrence. The aim of this study was to evaluate the correlation between the expression of Ki67, VEGF, and p53 in HCC and clinicopathological characteristics of HCC patients, as well as their predictive value for HCC recurrence after OLT. Methods. 60 patients who underwent OLT and were found to have HCC in the liver explant. The expression of Ki67, VEGF, and p53 in HCC was detected by immunohistochemistry. Results. Ki67 was associated with the tumor number and the grade of differentiation at baseline. VEGF was associated with the diameter and number of tumors, tumor differentiation, and lymph node metastasis. p53 was associated with the tumor diameter and tumor encapsulation. The expression of Ki67, VEGF, and p53 in HCC was correlated with the tumor recurrence after OLT, respectively. Among them, VEGF was an independent predictor for tumor recurrence after OLT. Conclusion. Ki67, VEGF, and p53 are associated with the recurrence of HCC after OLT. VEGF independently predicts the recurrence of HCC.
\end{abstract}

\section{Introduction}

Hepatocellular carcinoma (HCC) is one of the leading causes of cancer-related death globally $[1,2]$. According to statistics, the annual mortality of HCC in China accounts for $55 \%$ of the global one. Because the initial symptoms of HCC are not evident, the patients are often in the intermediate or advanced stages at diagnosis. The survival of untreated HCC patients after diagnosis is usually less than 6 months $[3,4]$.

To date, orthotopic liver transplantation (OLT) is one of the best options in case of end-stage liver disease [5]. With the advances in medicinal treatment, five-year survival rates after OLT of over $75 \%$ have been widely observed $[6,7]$.
Although the survival rate is relatively high, the risk of recurrence is the major concern in transplanted patients. Clinical factors that are related to the recurrence after OLT include the size and number of tumors, micro/macrovascular invasion, and high levels of serum alpha-fetoprotein (AFP) [8].

Tumor-related factors including Ki67, VEGF, and p53 have been reported to play a role in the development and progression of HCC [9-11].

Tumor proliferating antigen (Ki67) is a nuclear protein that is recognized as a sensitive marker for cell proliferation [11]. Ki67 is highly expressed in numerous human solid tumors and is correlated with patient prognosis. Several studies found that the expression of Ki67 in numerous cancers 
including prostate cancer, lung cancer, and HCC was negatively correlated with the therapeutic efficacy and prognosis [12-14]. However, the predictive value of Ki67 in the recurrence of HCC after OLT remains unclear.

Vascular endothelial growth factor (VEGF) is an important factor that mediates angiogenesis. It plays an indispensable role in tumor growth, invasion, and metastasis, as well as patient prognosis [15]. Nagoshi showed that VEGF promoted the proliferation of tumor vascular endothelial cells and served as an early marker of angiogenesis in HCC [16]. In a study of human OLT, it was suggested that the high recurrence rate after transplantation was associated with the expression of VEGF in liver grafts during the acute rejection phase [17]. Although high expression of VEGF promoted the recurrence of HCC after transplantation, whether VEGF predicts the recurrence remains unclear.

p53 is a tumor suppressor gene that regulates cell cycle and apoptosis [9]. Its mutations were found to be tumorigenic in several cancers including colon and prostate cancers, and HCC [18-20]. A study showed that the rates of gene mutation and upregulated expression of $p 53$ in HCC patients were $31.5 \%$ and $35.0 \%$, respectively, and the expression of p53 was significantly associated with the poor prognosis of HCC patients [21]. However, whether p53 is associated with the recurrence of HCC after OLT has not been revealed.

Detection of factors that are associated with HCC recurrence may predict the tumor recurrence in HCC patients after OLT. In this study, we detected the expression of Ki67, VEGF, and p53 in HCC by immunohistochemical staining and explored their predictive value in tumor recurrence after OLT.

\section{Materials and Method}

2.1. Study Population Clinicopathological Characteristics. The study population included 60 patients who had undergone OLT at Dongfang Hospital and had complete follow-up data. All patients were diagnosed of HCC before OLT. Recurrent HCC were confirmed by a liver biopsy and pathological examination.

2.2. Clinicopathological Parameters of HCC Patients. The characteristics of HCC patients including the Child-Pugh class, the diameter and number of tumors, serum level of AFP, TNM stage, vascular invasion, liver cirrhosis, tumor encapsulation, lymph node metastasis, and tumor differentiation were collected. Patients with recurrence of HCC within 2 years were divided into the recurrence group $(n=37)$. Patients whose HCC recurred after 2 years or did not recur until the last visit were divided into the control group $(n=23)$.

2.3. Immunohistochemical Staining. Five- $\mu \mathrm{m}$ slices were obtained from paraffin-embedded specimens of tumor. Sections were dewaxed in xylene and rehydrated in alcohol followed by wet autoclave pretreatment (10 minutes at $120^{\circ} \mathrm{C}$ ) in citrate buffer for antigen retrieval. These were rinsed in phosphate-buffered saline. Immunohistochemical staining for antibodies to Ki67 (Cat: ab15580, Abcam, Cambridge, MA), VEGF (Cat: 19003-1-AP, Proteintech, Chicago,
USA), and p53 (Cat: ab1101, Abcam, Cambridge, MA) was performed using the avidin-biotin-peroxidase complex method. The primary antibody was applied to the sections and allowed to react for $25 \mathrm{~min}$ at room temperature. The sections were then incubated with biotinylated anti-mouse/rabbit antibody (1:100 dilution for Ki67, VEGF, and p53) for $25 \mathrm{~min}$ and avidin-biotin-peroxidase reagent for $25 \mathrm{~min}$. After color development with diaminobenzimide, the sections were counterstained with hematoxylin.

2.4. Statistical Analysis. The correlation between Ki67, VEGF, and p53 and clinicopathological characteristics were analyzed by the $\chi^{2}$ test and Fisher test. The correlation between Ki67, VEGF, and p53 and tumor recurrence after OLT were analyzed by single factor survival analysis and COX multivariate regression. A $P$ value of $<0.05$ was considered statistically significant. All statistical analyses were performed using the SPSS 19.0 analysis software.

\section{Results}

The patients were three women and 57 men with a mean age of $55 \pm 15$ years. 54 patients were positive for hepatitis B surface antigen (HBsAg), and six were negative. 31 patients were with a tumor diameter of $<5 \mathrm{~cm}$, and 29 were of $5-15 \mathrm{~cm}$. The AFP level was $\geq 400 \mu \mathrm{g} / \mathrm{L}$ in 20 patients and $<400 \mu \mathrm{g} / \mathrm{L}$ in 40 . Postoperative pathological examination showed 11 cases of poorly differentiated HCC, 37 of moderately differentiated HCC, and 12 of well-differentiated HCC. The recurrence of tumors included eight cases of intrahepatic recurrence, seven of intrahepatic and extrahepatic recurrence, 18 of lung metastasis, nine of bone metastasis, six of lymph node metastasis, and three of brain metastasis.

3.1. Correlationship between Ki67, VEGF, and p53 and Clinicopathological Characteristics of HCC Patients. The expression of Ki67 was correlated with the number of tumors $(P=0.005)$ and the grade of tumor differentiation $(P=0.038)$. However, it was not associated with other clinicopathological characteristics of HCC patients (Table 1). The expression of VEGF was associated with the diameter and number of tumors $(P=0.037$ and $P=0.005)$, tumor differentiation $(P=0.035)$, and lymph node metastasis $(P=0.025)$, whereas it was not related to other clinicopathological characteristics (Table 1). The expression of p53 was correlated with the tumor diameter $(P=0.044)$ and tumor encapsulation $(P=0.022)$. There was no correlation between $\mathrm{p} 53$ and other clinicopathological characteristics (Table 1).

3.2. The Expression of Ki67, VEGF, and p53 in HCC. In the postoperative HCC samples from 37 patients in the recurrence group and 23 in the control group, the positive expression rates of Ki67 were 67.5\% (25/37) and 39.1\% (9/23), respectively (Figures 1(a), 1(b), and 2). The positive expression rates of VEGF were $56.7 \%(21 / 37)$ and $30.4 \%(7 / 23)$, respectively (Figures 3(a), 3(b), and 2). The positive expression rates of p53 were $62.1 \%(23 / 37)$ and $34.7 \%(8 / 23)$, respectively (Figures 4(a), 4(b), and 2). Logistic singlefactor statistical analysis showed that the positive expression rates of Ki67, VEGF, and p53 were different between the 
TABLE 1: Correlation between the expression of Ki67, VEGF, and p53 and clinicopathological characteristics of HCC patients.

\begin{tabular}{|c|c|c|c|c|c|c|c|c|c|c|}
\hline \multirow{2}{*}{\multicolumn{2}{|c|}{ Clinicopathological characteristics }} & \multicolumn{3}{|c|}{ Ki67 expression } & \multicolumn{3}{|c|}{ VEGF expression } & \multicolumn{3}{|c|}{ p53 expression } \\
\hline & & - & + & $P$ & - & + & $P$ & - & + & $P$ \\
\hline \multirow{3}{*}{ Gender } & & & & $1.000^{*}$ & & & $1.000^{*}$ & & & $0.238^{*}$ \\
\hline & Male & 25 & 32 & & 30 & 27 & & 29 & 28 & \\
\hline & Female & 2 & 1 & & 2 & 1 & & 0 & 3 & \\
\hline \multirow{3}{*}{ Age } & & & & $0.388^{*}$ & & & $0.088^{*}$ & & & $1.000^{*}$ \\
\hline & $\leqq 60$ & 22 & 32 & & 31 & 23 & & 26 & 28 & \\
\hline & $>60$ & 4 & 2 & & 1 & 5 & & 3 & 3 & \\
\hline \multirow{4}{*}{ Child-Pugh class } & & & & $1.000^{*}$ & & & $0.883^{*}$ & & & $0.494^{*}$ \\
\hline & A & 8 & 11 & & 10 & 9 & & 8 & 11 & \\
\hline & $\mathrm{B}$ & 18 & 12 & & 22 & 18 & & 21 & 19 & \\
\hline & $\mathrm{C}$ & 0 & 1 & & 0 & 1 & & 0 & 1 & \\
\hline \multirow{3}{*}{ Tumor size } & & & & $0.203^{\dagger}$ & & & $0.037^{\dagger}$ & & & $0.044^{\dagger}$ \\
\hline & $\leqq 5.0$ & 16 & 15 & & 21 & 10 & & 19 & 12 & \\
\hline & $>5.0$ & 10 & 19 & & 11 & 18 & & 10 & 19 & \\
\hline \multirow{3}{*}{ Tumor number } & & & & $0.005^{\dagger}$ & & & $0.005^{\dagger}$ & & & $0.617^{\dagger}$ \\
\hline & 1 & 19 & 12 & & 11 & 20 & & 16 & 15 & \\
\hline & $\geqq 2$ & 7 & 22 & & 21 & 8 & & 13 & 16 & \\
\hline \multirow{3}{*}{ AFP } & & & & $0.174^{\dagger}$ & & & $1.000^{\dagger}$ & & & $0.419^{\dagger}$ \\
\hline & $>400$ & 6 & 14 & & 11 & 9 & & 8 & 12 & \\
\hline & $\leqq 400$ & 20 & 20 & & 21 & 19 & & 21 & 19 & \\
\hline \multirow{5}{*}{ TNM staging } & & & & $0.122^{*}$ & & & $0.659^{*}$ & & & $0.857^{*}$ \\
\hline & I & 4 & 2 & & 4 & 2 & & 3 & 3 & \\
\hline & II & 11 & 9 & & 12 & 8 & & 4 & 11 & \\
\hline & III & 6 & 3 & & 3 & 6 & & 9 & 5 & \\
\hline & IV & 5 & 20 & & 13 & 12 & & 13 & 12 & \\
\hline \multirow{3}{*}{ Vascular invasion } & & & & $0.398^{*}$ & & & $0.783^{\dagger}$ & & & $0.544^{\dagger}$ \\
\hline & Yes & 4 & 14 & & 6 & 12 & & 7 & 11 & \\
\hline & No & 22 & 20 & & 26 & 16 & & 22 & 20 & \\
\hline \multirow{3}{*}{ Cirrhosis } & & & & $0.072^{*}$ & & & $0.379^{\dagger}$ & & & $0.082^{*}$ \\
\hline & Yes & 23 & 23 & & 23 & 23 & & 25 & 21 & \\
\hline & No & 3 & 11 & & 9 & 5 & & 4 & 10 & \\
\hline \multirow{3}{*}{ Tumor encapsulation } & & & & $0.416^{\dagger}$ & & & $0.578^{\dagger}$ & & & $0.022^{*}$ \\
\hline & Yes & 21 & 21 & & 24 & 19 & & 25 & 18 & \\
\hline & No & 5 & 12 & & 8 & 9 & & 4 & 13 & \\
\hline \multirow{3}{*}{ Lymphatic metastasis } & & & & $1.000^{*}$ & & & $0.025^{*}$ & & & $0.750^{*}$ \\
\hline & Yes & 1 & 1 & & 0 & 2 & & 0 & 2 & \\
\hline & No & 25 & 33 & & 32 & 26 & & 29 & 29 & \\
\hline \multirow{4}{*}{ Differentiation } & & & & $0.038^{*}$ & & & $0.035^{*}$ & & & $0.081^{*}$ \\
\hline & Low & 1 & 10 & & 2 & 9 & & 2 & 9 & \\
\hline & Medium & 19 & 18 & & 22 & 15 & & 21 & 37 & \\
\hline & High & 6 & 6 & & 8 & 4 & & 6 & 12 & \\
\hline
\end{tabular}

*: Fisher test; †: Pearson chi-square test; “+”: positive; “-”: negative.

recurrence group and the control group, respectively $(P=0.015,0.008$, and 0.035 , Table 2$)$.

3.3. Correlationship between the Expression of Ki67, VEGF, and $p 53$ and the Recurrence of HCC after OLT. Single-factor survival analysis showed that the positive expression of Ki67
(Log Rank $P=0.036$ and Breslow $P=0.047$ ), the positive expression of $\operatorname{VEGF}(P=0.003$ and $P=0.001)$, and the positive expression of p53 $(P=0.015$ and $P=0.011)$ were associated with tumor recurrence, respectively (Table 3, Figures 5(a)-5(c)).

As analyzed by multivariate regression of COX, the diameter and number of tumors $(P=0.016)$, the tumor 


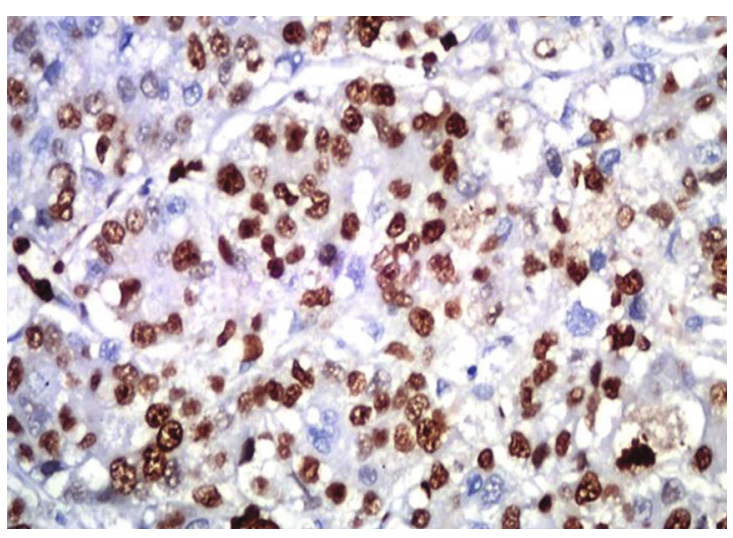

(a)

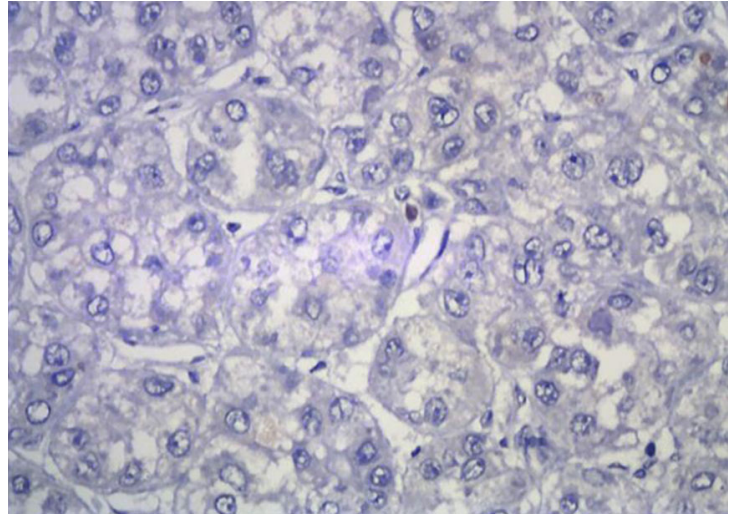

(b)

Figure 1: (a) Positive immunostaining with nuclear Ki67 expression in HCC. (b) Negative Ki67 expression with low nuclear reactivity in HCC.

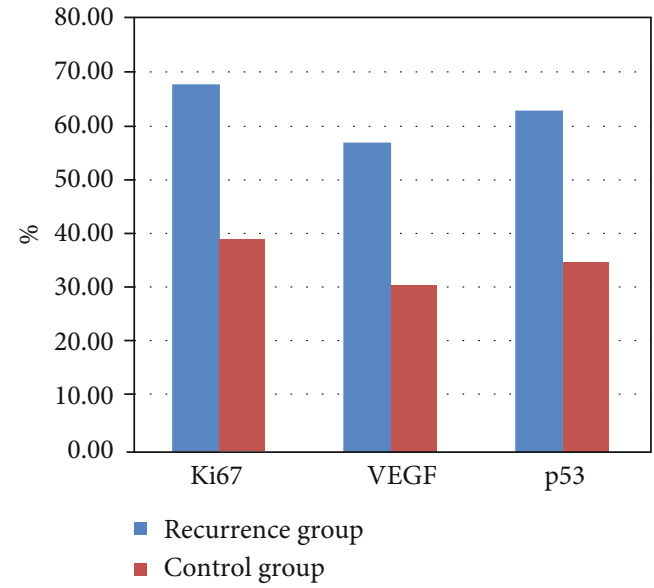

Figure 2: The positive expression rates of Ki67, VEGF, and p53 in the recurrence group and the control group.

encapsulation $(P=0.022)$, the vascular invasion $(P=0.009)$, the TNM stage $(P=0.036)$, and the positive expression of VEGF $(P=0.005)$ in HCC were associated with the HCC recurrence after OLT. However, the positive expression of Ki67 $(P=0.142)$ and p53 $(P=0.062)$ did not predict the tumor recurrence and metastasis (Table 4$)$.

\section{Discussion}

The recurrence of HCC gives rise to an unsatisfactory survival rate of HCC patients after OLT. Several studies suggested that the causes of HCC recurrence after OLT might include the following [22-25]: (1) Micrometastasis of cancer cells had occurred before transplantation. (2) Handling the diseased liver during the operation caused tumor rupture and thereby iatrogenic cancer metastasis. (3) The use of immunosuppressants promoted the proliferation and invasion of tumor cells. Recent studies showed that tumor cells and several biological molecules secreted by the microenvironment of tumors play an important role in the recurrence of tumors [26]. Preoperative detection of these molecular markers and postoperative quantitative assays of corresponding antibodies may predict tumor recurrence after OLT.

The current study showed that the expression of Ki67 in HCC was correlated with the number of tumors and the grade of tumor differentiation in HCC patients whereas it was not associated with other clinicopathological characteristics. It is suggested that the expression of Ki67 is related to the proliferation and malignant biological activities of liver cancer cells. In addition, the positive expression rate of Ki67 was higher in the recurrence group than the control group. The patients with positive expression of $\mathrm{Ki} 67$ had worse disease-free survival after surgery. It is suggested that HCC with high expression of $\mathrm{Ki} 67$ is prone to invasion and metastasis. However, COX multivariate survival analysis indicated that Ki67 had no independent predictive value for tumor recurrence after OLT. We infer that $\mathrm{Ki} 67$ is not specific to malignant tumors, and its expression may be affected by other factors, such as nutrient supply to cells. Therefore, Ki67 may be useful in predicting the prognosis when combined with other indicators.

VEGF is an essential factor in tumor growth, which plays a role in tumor growth and invasion, and patients prognosis [17]. The increased level of VEGF is mainly secreted by an autocrine or a paracrine measure by hepatic stellate cells and tumor cells [25]. Jeng et al. found that the expression of VEGF mRNA in HCC patients with the portal vein tumor was significantly higher than those without, and multivariate analysis also showed that the expression of VEGF was correlated with the portal vein thrombosis [27]. In the current study, the expression of VEGF in HCC was correlated with the diameter and number of tumors, tumor differentiation, and lymph node metastasis, which is consistent with the study by Jia et al. [28]. It is indicated that VEGF is involved in the development and progression of HCC. In addition, the positive expression rate of VEGF in the recurrence group was higher than the control group and the expression of VEGF was negatively correlated with the tumor-free survival. Therefore, VEGF is a critical risk factor for HCC recurrence after OLT and is an independent predictor of tumor recurrence after OLT in HCC patients. 


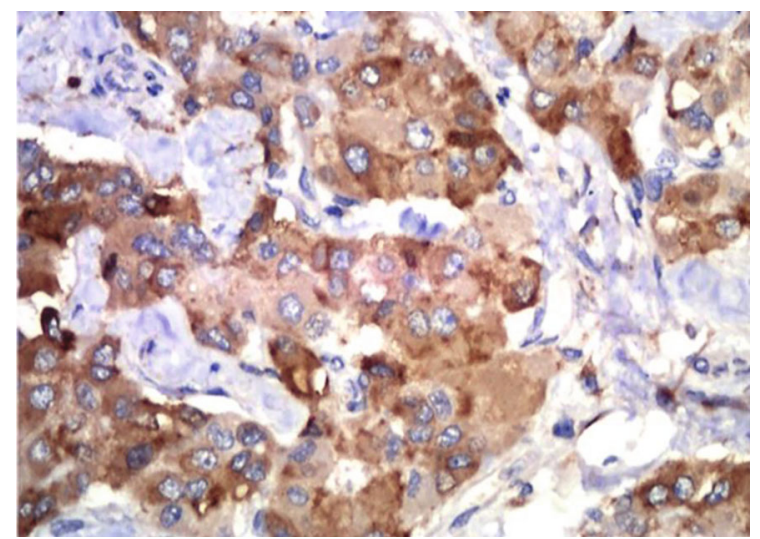

(a)

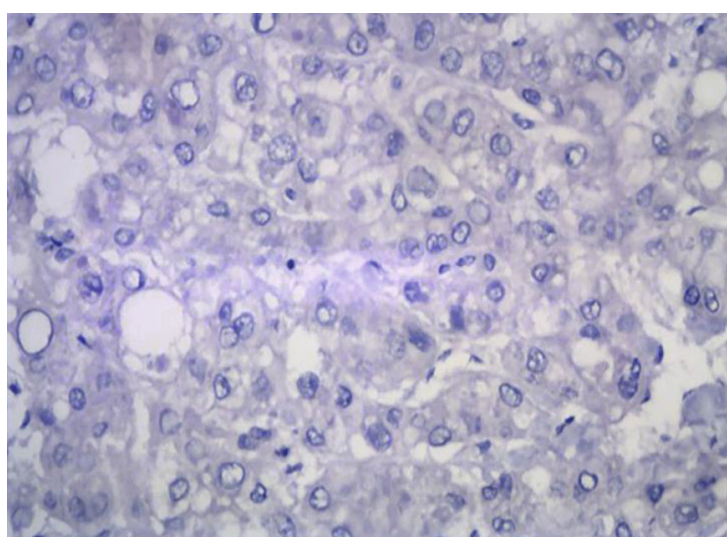

(b)

Figure 3: (a) Positive immunostaining with VEGF expression in HCC. (b) Negative VEGF expression immunostaining in HCC.

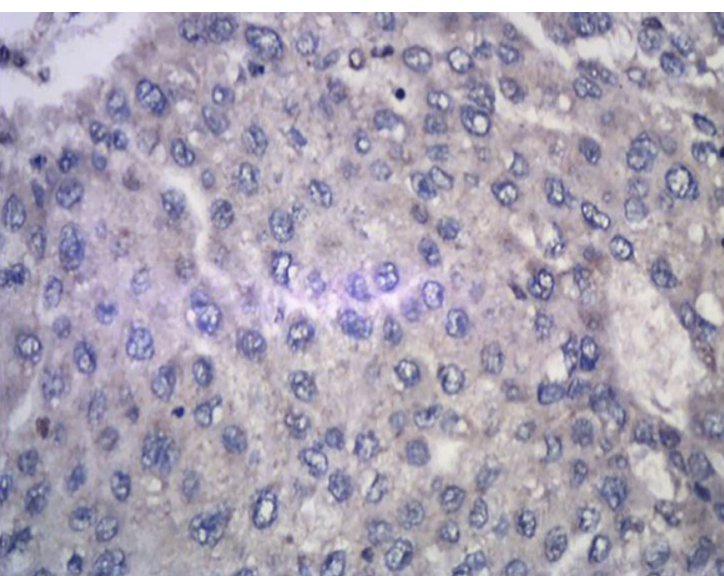

(a)

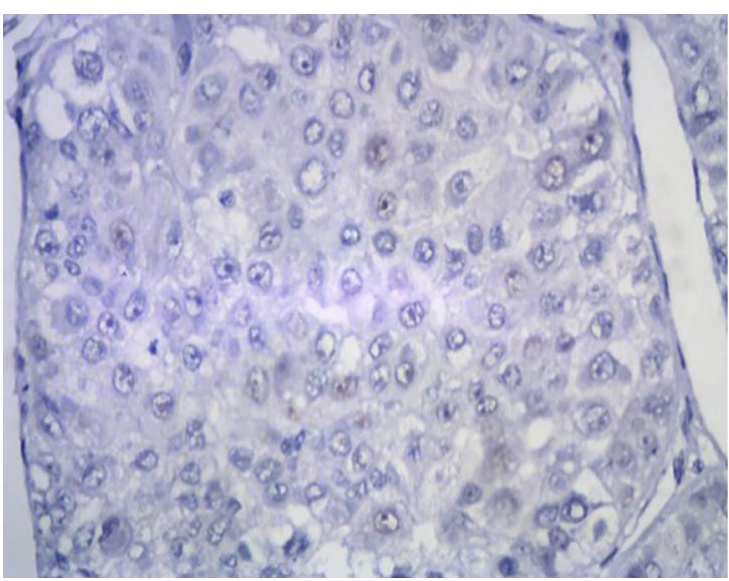

(b)

Figure 4: (a) Positive immunostaining with p53 expression in HCC. (b) Negative p53 expression immunostaining in HCC.

TABLE 2: The expression of Ki67, VEGF, and p53 in the recurrence group and the control groups.

\begin{tabular}{lcccc}
\hline Name & Expression & Relapse group & Control group & $95 \% \mathrm{CI}^{*}$ \\
\hline \multirow{2}{*}{ Ki67 } & Negative & 12 & 14 & $(0.288,3.718)$ \\
& Positive & 25 & 9 & 0.015 \\
\multirow{2}{*}{ VEGF } & Negative & 16 & 16 & $(0.404,3.588)$ \\
& Positive & 21 & 7 & 0.008 \\
p53 & Negative & 14 & 15 & $(0.043,2.823)$ \\
& Positive & 23 & 14 & 0.035 \\
\hline
\end{tabular}

*: logistic analysis.

TABLE 3: Correlation between Ki67, VEGF, and p53 expression and tumor recurrence.

\begin{tabular}{lcccc}
\hline Name & $\chi^{2}$ & Log Rank $P$ value & $\chi^{2}$ & Breslow $P$ value \\
\hline Ki67 & 4.404 & 0.036 & 3.936 & 0.047 \\
VEGF & 8.807 & 0.003 & 11.614 & 0.001 \\
p53 & 5.947 & 0.015 & 6.445 & 0.011 \\
\hline
\end{tabular}

Note: Kaplan-Meier analysis with two analytical methods. 


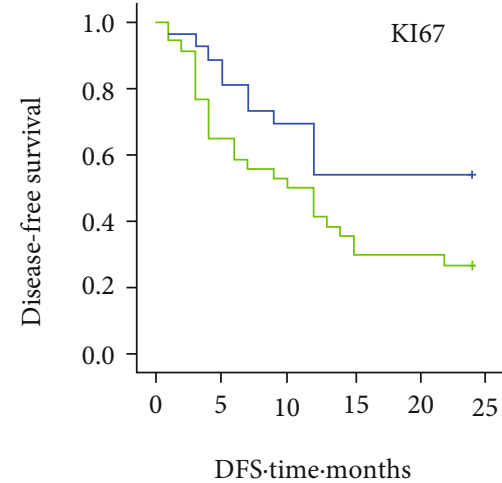

(a)

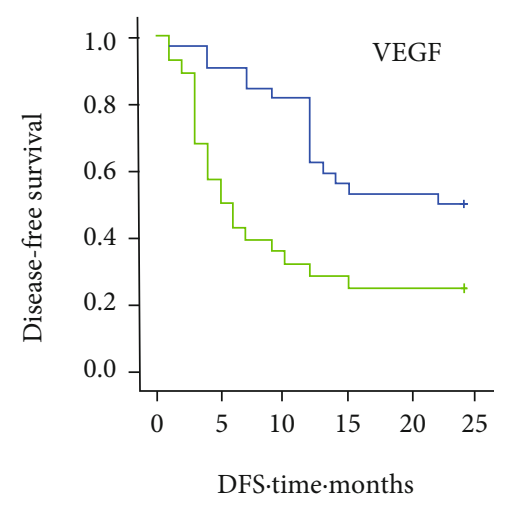

(b)

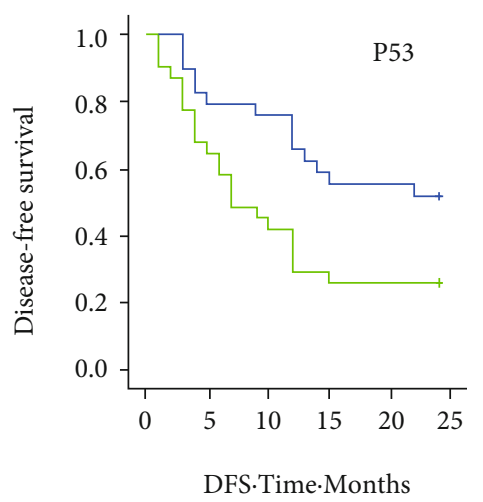

(c)

FIGURE 5: (a) Disease-free survival (DFS) of patients with negative and positive expression of Ki67 expression; blue line: negative; light green line: positive; $\log$ Rank $P=0.036$; Breslow $P=0.047$; (b) disease-free survival (DFS) of patients with negative and positive expression of VEGF. Blue line: negative; light green line: positive; Log Rank $P=0.003$; Breslow $P=0.001$. (c) Disease-free survival (DFS) of patients with negative and positive expression of $\mathrm{p} 53$, Log Rank $P=0.015$; Breslow $P=0.011$.

TABLE 4: Correlation between the expression of Ki67, VEGF, and p53 and recurrence of HCC after OLT.

\begin{tabular}{lcccccr}
\hline & $\beta$ & S.E & Wald $\chi^{2}$ & $P$ value & OR & 95\% CI* \\
\hline Ki67 & 0.642 & 0.438 & 2.153 & 0.142 & 1.901 & $(-0.513,1.872)$ \\
VEGF & 1.387 & 0.492 & 7.942 & 0.005 & 4.004 & $(0.346,3.265)$ \\
p53 & 0.801 & 0.430 & 3.474 & 0.062 & 2.228 & $(-0.147,2.101)$ \\
\hline
\end{tabular}

*: COX multifactor regression analysis.

In this study, p53 expression was associated with tumor encapsulation and tumor diameter, but not with the age, serum AFP level, number of tumors, tumor differentiation, and TNM stage $(P>0.05)$. It indicates that the proliferation and invasion of liver cancer cells after OLT are related to the expression of p53. Additionally, the positive expression rate of p53 was high in the recurrence group and it was associated with tumor recurrence. However, it was showed that the positive expression of p53 did not exhibit predictive value in tumor recurrence after OLT in HCC patients.

There were several limitations regarding the study. It was a retrospective study and had a relatively small sample. Only the correlationship between markers and clinicopathological characteristics were demonstrated but no in-depth mechanisms were revealed. Future studies with a larger cohort of patient samples will be needed to further support the findings.

\section{Conclusion}

In conclusion, Ki67, VEGF, and p53 are associated with the recurrence of HCC patients after OLT. Nevertheless, only VEGF independently predicts the recurrence of HCC patients after OLT. It is necessary to identify robust predictors of HCC recurrence after OLT, which facilitates the screening of patients with a high risk of HCC recurrence.

\section{Data Availability}

The data used to support the findings of this study are available from the corresponding authors upon request.

\section{Conflicts of Interest}

The authors declare that they have no known competing financial interests or personal relationships that could have appeared to influence the work reported in this paper.

\section{Authors' Contributions}

Xia Zhang and Zhixian Wu contributed equally to this work.

\section{Acknowledgments}

This work was supported by the Fujian Provincial Social Development Guiding Key Project (grant number 2016Y0068), Science and Technology Innovation Joint Fund Project of Fujian Province (grant number 2019Y9044), and Natural Science Foundation of Fujian Province (grant number 2020J011131). The authors would like to thank Lili Zhou and Fengsui Chen for the support in this work (HospitalLevel Funds of 900th Hospital: grant number 2020L25, and 2018Q07).

\section{References}

[1] A. Jemal, F. Bray, M. M. Center, J. Ferlay, E. Ward, and D. Forman, "Global cancer statistics," CA: a Cancer Journal for Clinicians, vol. 61, no. 2, pp. 69-90, 2011.

[2] J. D. Yang and L. R. Roberts, "Hepatocellular carcinoma: a global view," Nature Reviews. Gastroenterology \& Hepatology, vol. 7, no. 8, pp. 448-458, 2010.

[3] J. Ferlay, H. R. Shin, F. Bray, D. Forman, C. Mathers, and D. M. Parkin, "Estimates of worldwide burden of cancer in 2008: 
GLOBOCAN 2008," International Journal of Cancer, vol. 127, no. 12, pp. 2893-2917, 2010.

[4] D. M. Parkin, F. Bray, J. Ferlay, and P. Pisani, "Estimating the world cancer burden: Globocan 2000," International Journal of Cancer, vol. 94, no. 2, pp. 153-156, 2001.

[5] T. E. Starzl, C. G. Groth, L. Brettschneider et al., "Orthotopic homotransplantation of the human liver," Annals of Surgery, vol. 168, no. 3, pp. 392-415, 1968.

[6] C. L. Chen, S. T. Fan, S. G. Lee, M. Makuuchi, and K. Tanaka, "Living-donor liver transplantation: 12 years of experience in Asia," Transplantation, vol. 75, Supplement, pp. S6-S11, 2003.

[7] C. S. Ahn, S. Hwang, K. H. Kim et al., "Long-term outcome of living donor liver transplantation for patients with alcoholic liver disease," Transplantation Proceedings, vol. 46, no. 3, pp. 761-766, 2014.

[8] K. Nakanishi, M. Sakamoto, S. Yamasaki, S. Todo, and S. Hirohashi, "Akt phosphorylation is a risk factor for early disease recurrence and poor prognosis in hepatocellular carcinoma," Cancer, vol. 103, no. 2, pp. 307-312, 2005.

[9] J. Liu, Q. Ma, M. Zhang et al., "Alterations of TP53 are associated with a poor outcome for patients with hepatocellular carcinoma: evidence from a systematic review and meta-analysis," European Journal of Cancer, vol. 48, no. 15, pp. 2328-2338, 2012.

[10] D. F. Yao, L. W. Qiu, W. Wu, N. H. Yao, S. S. Li, and Y. Z. Bian, "Dynamic alterations of VEGF and intervention of its expression on effect of hepatocyte malignant transformation," Zhonghua Yi Xue Za Zhi, vol. 90, no. 42, pp. 3014-3018, 2010.

[11] T. Scholzen and J. Gerdes, "The Ki-67 protein: from the known and the unknown," Journal of Cellular Physiology, vol. 182, no. 3, pp. 311-322, 2000.

[12] L. Bubendorf, C. Tapia, T. C. Gasser et al., "Ki67 labeling index in core needle biopsies independently predicts tumor- specific survival in prostate cancer," Human Pathology, vol. 29, no. 9, pp. 949-954, 1998.

[13] B. Martin, M. Paesmans, C. Mascaux et al., "Ki-67 expression and patients survival in lung cancer: systematic review of the literature with meta-analysis," British Journal of Cancer, vol. 91, no. 12, pp. 2018-2025, 2004.

[14] I. O. Ng, J. Na, E. C. Lai, S. T. Fan, and M. Ng, "Ki-67 antigen expression in hepatocellular carcinoma using monoclonal antibody MIB1. A comparison with proliferating cell nuclear antigen," American Journal of Clinical Pathology, vol. 104, no. 3, pp. 313-318, 1995.

[15] A. X. Zhu, D. G. Duda, D. V. Sahani, and R. K. Jain, "HCC and angiogenesis: possible targets and future directions," Nature Reviews. Clinical Oncology, vol. 8, no. 5, pp. 292-301, 2011.

[16] S. Nagoshi, "Osteopontin: versatile modulator of liver diseases," Hepatology Research, vol. 44, no. 1, pp. 22-30, 2014.

[17] B. Deng, X. F. Zhang, X. C. Zhu et al., "Correlation and prognostic value of osteopontin and Bcl-2 in hepatocellular carcinoma patients after curative resection," Oncology Reports, vol. 30, no. 6, pp. 2795-2803, 2013.

[18] M. Hollstein, D. Sidransky, B. Vogelstein, and C. C. Harris, "p53 mutations in human cancers," Science, vol. 253, no. 5015 , pp. 49-53, 1991.

[19] S. J. Baker, E. R. Fearon, J. M. Nigro et al., "Chromosome 17 deletions and p53 gene mutations in colorectal carcinomas," Science, vol. 244, no. 4901, pp. 217-221, 1989.

[20] I. C. Hsu, R. A. Metcalf, T. Sun, J. A. Welsh, N. J. Wang, and C. C. Harris, "Mutational hot spot in the p53 gene in human hepatocellular carcinomas," Nature, vol. 350, no. 6317, pp. 427-428, 1991.

[21] J. Cheng, W. Wang, C. Sun, M. Li, B. Wang, and Y. Lv, "Metaanalysis of the prognostic and diagnostic significance of serum/plasma osteopontin in hepatocellular carcinoma," Journal of Clinical Gastroenterology, vol. 48, no. 9, pp. 806-814, 2014.

[22] J. Fuster, R. Charco, J. M. Llovet, J. Bruix, and J. C. Garcia-Valdecasas, "Liver transplantation in hepatocellular carcinoma," Transplant International, vol. 18, no. 3, pp. 278-282, 2005.

[23] H. K. Ko, G. Y. Ko, H. K. Yoon, and K. B. Sung, "Tumor response to transcatheter arterial chemoembolization in recurrent hepatocellular carcinoma after living donor liver transplantation," Korean Journal of Radiology, vol. 8, no. 4, pp. 320-327, 2007.

[24] M. Fiorentino, A. Altimari, M. Ravaioli et al., "Predictive value of biological markers for hepatocellular carcinoma patients treated with orthotopic liver transplantation," Clinical Cancer Research, vol. 10, no. 5, pp. 1789-1795, 2004.

[25] C. de Vries, J. A. Escobedo, H. Ueno, K. Houck, N. Ferrara, and L. T. Williams, "The fms-like tyrosine kinase, a receptor for vascular endothelial growth factor," Science, vol. 255, no. 5047, pp. 989-991, 1992.

[26] H. L. Chen, H. Y. OuYang, Y. Le et al., “Aberrant MCT4 and GLUT1 expression is correlated with early recurrence and poor prognosis of hepatocellular carcinoma after hepatectomy," Cancer Medicine, vol. 7, no. 11, pp. 5339-5350, 2018.

[27] K. S. Jeng, I. S. Sheen, Y. C. Wang et al., "Is the vascular endothelial growth factor messenger RNA expression in resectable hepatocellular carcinoma of prognostic value after resection?," World Journal of Gastroenterology, vol. 10, no. 5, pp. 676-681, 2004.

[28] Z. Z. Jia, G. M. Jiang, and Y. L. Feng, "Serum HIF-1alpha and VEGF levels pre- and post-TACE in patients with primary liver cancer," Chinese Medical Sciences Journal, vol. 26, no. 3, pp. 158-162, 2011. 